ARTICLE

\title{
Development of the Efficient Modeling Method with Complicated Human Geometry for Monte-Carlo Treatment Planning System
}

\author{
Hiroaki KUMADA ${ }^{1, *}$, Kimiaki SAITO2 ${ }^{2}$, Takeji SAKAE ${ }^{1}$, \\ Akira MATSUMURA $^{1}$, Takemi NAKAMURA ${ }^{2}$ and Koji $\mathrm{ONO}^{3}$ \\ ${ }^{1}$ University of Tsukuba, 1-1-1 Tennodai, Tsukuba-shi, Ibaraki-ken, 305-8575, Japan \\ ${ }^{2}$ Japan Atomic Energy Agency, 2-4 Shirakata, Tokai-mura, Naka-gun, Ibaraki-ken, 319-1195, Japan \\ ${ }^{3}$ Kyoto University Research Reactor Institute, 2, Asashiro-Nishi, Kumatori-cho,Sennan-gun, Osaka-fu,590-0494, Japan
}

\begin{abstract}
A new multi-modal Monte-Carlo treatment planning system (developing code: JCDS-FX) is being developed to implement accurate treatment planning for boron neutron capture therapy. JCDS-FX employs multi-purpose Monte-Carlo code: PHITS as dose calculation engine. For calculation of dose distribution for a human body, JCDS-FX can make a precise voxel model consisting of pixel based voxel cells like $0.4 \times 0.4 \times 2.0 \mathrm{~mm}^{3}$ voxel in order to perform high-accuracy dose estimation. However, the miniaturization of the voxel size causes calculation time to increase. The aim of this study is to investigate sophisticated modeling method which can perform Monte-Carlo calculation for human geometry efficiently. Thus, we devised a new voxel modeling method "Multistep lattice-voxel method" which can configure an efficient voxel model that combines different voxel sizes by utilizing the lattice function over and over.

To verify the performance of the calculation with the modeling method, several calculations for human geometry were carried out. The verification results proved that the method enabled to reduce calculation time substantially while keeping the high-accuracy dose estimation. Several verifications with complicated human geometry are being performed at present. And we are still carrying out sophistication of the modeling method to work toward practical use of the new treatment planning system with more precise computational dosimetry.
\end{abstract}

KEYWORDS: treatment planning, Monte-Carlo, boron neutron capture therapy, particle therapy

\section{Introduction}

Boron neutron capture therapy (BNCT) is a sort of particle radiation therapy for obstinate cancer such as malignant brain tumors and melanoma. ${ }^{1)}$ The physical principle of the BNCT was presented in 1936 and its medical application for malignant brain tumors was conducted. ${ }^{2)}$ Clinical trial of BNCT is being performed using some research reactors in world-wide. At present in Japan, clinical trials for not only brain tumor and melanoma but also for head-\&-neck cancer, liver cancer and lung cancer are being carried out. At Japan Research Reactor No.4(JRR-4) in Japan Atomic Energy Agency (JAEA), BNCT clinical trial for malignant brain tumor was begun using thermal neutron beam in $1999,{ }^{3,4)}$ and then clinical trials for head-\&-neck cancer, melanoma and pulmonary tumor were begun at JRR-4 in 2004. ${ }^{5}$ )

To carry out BNCT based on accurate treatment plan, JCDS as a Monte-Carlo based treatment planning system was developed by JAEA. ${ }^{6,7)}$ Development of JCDS enabled to obtain detail dose information in treatment planning of BNCT and to determine an optimum irradiation condition for a patient. JCDS was applied practically to the clinical trial of BNCT carried out at JRR-4 in 2003, and most of BNCT clinical trials in JRR-4 are performed based on treatment planning by JCDS.

*Corresponding author, E-mail: kumada@pmrc.tsukuba.ac.jp
In current BNCT studies in Japan, to further improve the BNCT outcome, various approaches such as combined modality therapy (X-ray therapy or proton radiotherapy boosted after BNCT), ${ }^{8}$ expansion of application of BNCT and accelerator based BNCT are being performed. The aim of the study for accelerator based BNCT is to develop compact neutron source with accelerator so that the BNCT can be performed in a hospital. However, to address these trials, we have to carry further developments for irradiation techniques and dosimetry method.

In University of Tsukuba, proton radiotherapy is being performed against various cancer patients at Proton Medical Research Center (PMRC) ${ }^{9}{ }^{9}$ In current treatment planning of the radiotherapy at not only PMRC but also most particle radiotherapy facilities, the discrete ordinate method (Sn method) like pencil beam method is applied to determine therapeutic dose and its distribution in a short time. However for the purpose of improvement of accuracy of the dose estimation in the treatment planning work and Quality Assurance/Quality Control of the radiotherapy, it is expected that treatment planning system with Monte-Carlo method is applied to the radiotherapy practically.

Based on the background, we are developing a new multi-modal Monte-Carlo treatment planning system builds on fundamental technologies of JCDS. ${ }^{10,11)}$ Several technologies and features of JCDS have been taken over to the new system (developing code: JCDS-FX). However, several new 


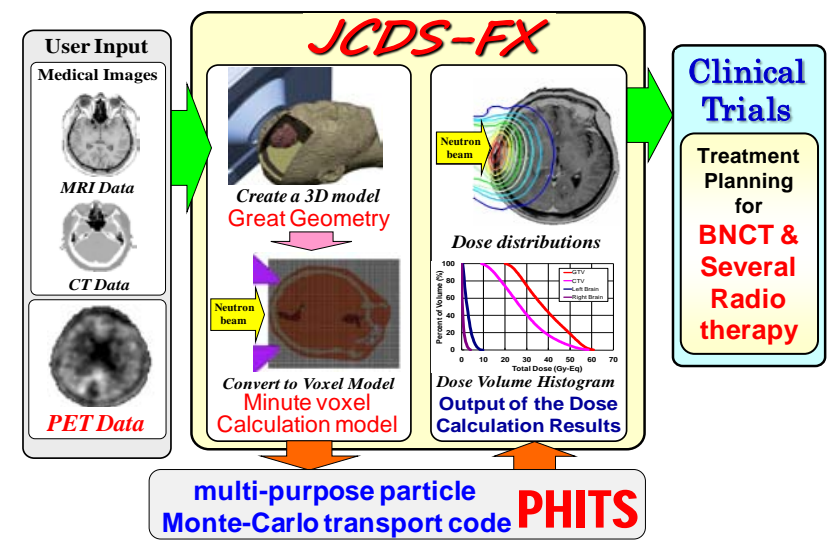

Fig. 1 Treatment planning procedure with JCDS-FX

technologies have been also installed to the system. Figure 1 shows schema of procedure of treatment planning with JCDS-FX.

In construction process of a three-dimensional patient model based on medical images, JCDS-FX can deal with PET images in addition to CT and MRI images. And in the process of creating a calculation model, concept of voxel modeling method which can construct a calculation model for complicated human body, has been inherited to JCDS-FX. The modeling method of JCDS-FX has been improved further; JCDS-FX allows to makes minute voxel model consisting of pixel based voxel cell in addition to the conventional $2 \times 2 \times 2 \mathrm{~mm}^{3}$ voxel of JCDS. Application of the miniature voxel model to dose estimation enables to perform higher accuracy computational dosimetry, the miniature voxel model works in particular estimation for mixed region between air and body tissue like nasal cavity.

However, the miniaturization of the voxel cell size causes calculation time to increase, because the number of the voxel cells constructing the geometry correlates with the efficiency of the Monte-Carlo particles transport. Therefore, at present, it is difficult to apply practically the dose calculation by the pixel based voxel model to treatment planning of BNCT.

Thus, the aim of this study is to investigate sophisticated modeling method which can perform Monte-Carlo calculations with human geometry efficiently. We devised an innovative voxel modeling method "Multistep Lattice-Voxel Method" which can configure an uneven voxel model that combines different voxel sizes by utilizing the lattice function over and over. To verify the performance of the modeling method, several calculations for human geometry were carried out.

\section{Material and Methods}

\section{Application of Multipurpose Monte-Carlo Code, PHITS}

One of the features of JCDS-FX is that PHITS is applied to the Monte-Carlo calculation engine, though JCDS employed MCNP. ${ }^{12)}$ PHITS, a multi-purpose particle Monte-Carlo transport code is under development by JAEA and some institutes. ${ }^{13)}$ PHITS can compute several particles behavior not only for neutrons and photons but also protons and heavy ions, thus PHITS allows determining doses generated by various radiotherapies as X-ray therapy, proton radiotherapy and BNCT. Therefore, application of PHITS to the dose calculation engine of treatment planning system enables to perform treatment planning of BNCT as well as particle radiotherapy. Moreover total dose given to a patient by a combined modality therapy like combination between BNCT and proton therapy can be also determined. In accelerator based BNCT study, dose accuracy is expected to improve since dose calculation including proper neutron source reaction between proton and target material can be carried out.

"Lattice" function installed in MCNP is installed to PHITS. The lattice function is a useful function as will be described later. The lattice function allows defining the complicated geometry like a human body easily. Hence, PHITS has been employed as dosimetry engine to JCDS-FX.

\section{Voxel Calculation Method Using Lattice Function}

To perform particle transport calculation with a human body, both JCDS and JCDS-FX create a voxel calculation model based on medical images. JCDS makes voxel model consisting of $5 \times 5 \times 5 \mathrm{~mm}^{3}$ or $2 \times 2 \times 2 \mathrm{~mm}^{3}$ voxel cells. On the other hand, JCDS-FX allows to makes pixel based voxel cell like $0.4 \times 0.4 \times 2 \mathrm{~mm}^{3}$ voxel cell in addition to the conventional $2 \times 2 \times 2 \mathrm{~mm}^{3}$ voxel, as described above. Both of the systems utilize the lattice function as a means to define the voxel model easily.

In conventional voxel model like $5 \times 5 \times 5 \mathrm{~mm}^{3}$ voxel cell model, material for individual voxel cells are defined based on the proportion of several tissues such as bone, soft tissue, and air, rounded off to the nearest $10 \%$ fraction by volume. Thus at boundary region, mixed material is applied to voxel cell of the region. For the pixel based voxel model by JCDS-FX, every voxel cell is defined by non-mixed material. For that reason, the application of the pixel based voxel model enable to improve accuracy of the dose estimation especially for boundary region.

\section{Multistep Lattice Voxel Method}

In the conventional modeling method with lattice function, a voxel model is configured by setting out voxel cells involving material information in order. The voxel cells are all in the same size. In case of the pixel based voxel model with the conventional modeling method, the number of the voxel cells is $262,144(512 \times 512)$ per slice. In contrast, by using the multistep lattice-voxel method (MLV method), the number of the voxel cells can be reduced dramatically, while keeping the accuracy of the calculation geometry. Figure 2 shows schema of the configuration of the voxel model with the MLV method.

The MLV method configures a voxel calculation model by the following procedure; first, a CT slice $(512 \times 512$ pixel $)$ is separated into $32 \times 32(1,024)$ regions, and each separated region consisting of $16 \times 16$ (256) pixels are grouped into a "large voxel region". When material in a large voxel region is uniform, the region is assigned to a "single large voxel" 


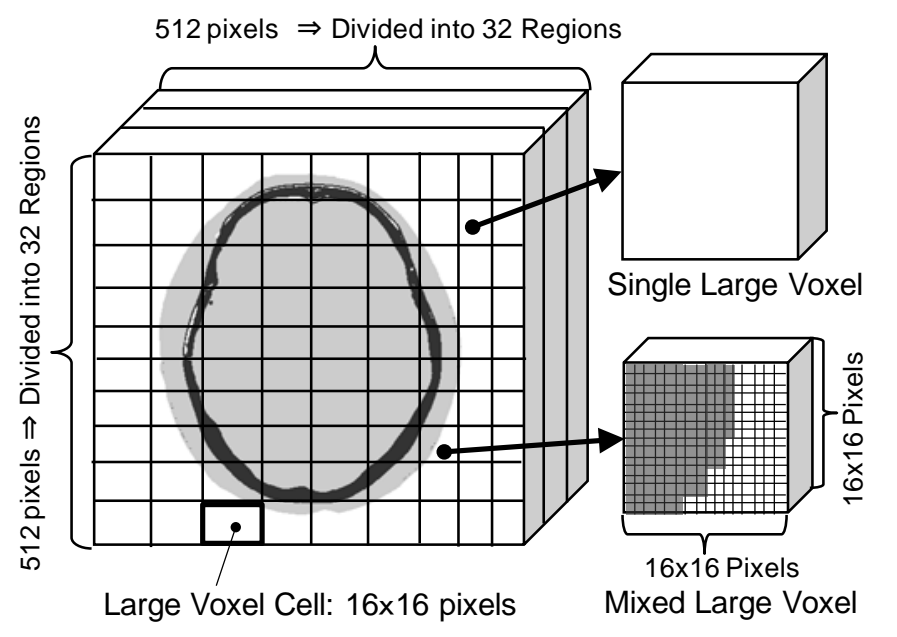

Fig. 2 Voxel model with the MLV method

cell. On the other hand, if two or more materials are mixed in the region, a "mixed large voxel” cell consisting of 256 miniature voxel cells is configured using the lattice function. The miniature voxel size equals pixel size. In accordance with the rule, every large voxel region of a slice is converted into the "single voxel cell" or "the mixed large voxel cell", and then uneven voxel model of a slice that combines different voxels sizes is made. This modeling procedure is applied to every slice, and then every slice consisting of the different voxels sizes is put in using the lattice function again. Finally, a three-dimensional voxel model is created. Number of the voxel cells of the uneven voxel model reduces substantially with the pixel based voxel model. Meanwhile the material composition of the uneven voxel model is exactly the same as the pixel based voxel model.

\section{Verification of the Multistep Lattice Voxel Method}

To verify characteristics and performance of the MLV method, several voxel models of a cylindrical water phantom were made, and then neutron flux distributions in the phantom for each voxel model were determined respectively. Finally, calculation results were compared.

(1) Phantom experiment at JRR-4

Neutron irradiation experiment with a cylindrical water phantom was carried out at JRR-4 in order to evaluate characteristics of the JRR-4's neutron beam for BNCT experimentally. Figure 3 shows schema of the phantom experiments. A circular beam outlet of $10 \mathrm{~cm}$ diameter was applied. The phantom of $18.6 \mathrm{~cm}$ in diameter and $24 \mathrm{~cm}$ in depth was made of $3 \mathrm{~mm}$ thick polymethylmethacrylate (PMMA) and filled with light water inside. In the experiments, the phantom was set at the front of the beam port in the irradiation room of JRR-4, gap between beam port and phantom's surface was $1 \mathrm{~cm}$. Then the phantom was irradiated by epithermal neutron beam which is being applied to clinical trials of BNCT. In the experiment, reactor power was $3,500 \mathrm{~kW}$ as peak rated power of JRR-4. In the phantom experiments, thermal neutron flux $(<0.53 \mathrm{eV})$ were determined by measuring the activities of the gold wires set in the phantom. To measure the distribution of thermal neutron

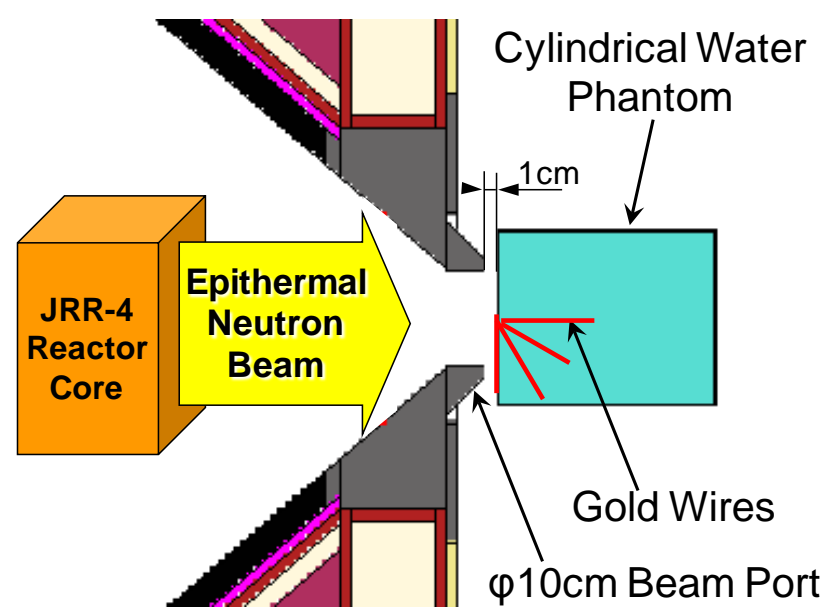

Fig. 3 Outline of the phantom experiment in JRR-4

flux in the phantom, bear gold wires or cadmium covered gold wires placed radially in the phantom had been irradiated in same beam condition respectively. The activations of the gold wires were measured with a simultaneous $\beta-\gamma$ coincidence counting device ( $\gamma$-detector: $3 \times 3$ in $\mathrm{NaI}(\mathrm{Tl})$ scintillator, $\beta$-detector: $2 \times 0.118$ in plastic scintillator). The thermal neutron flux was determined from the differences in the activation of each irradiated gold wire on same position. ${ }^{14)}$ Uncertainties of the measured thermal neutron flux value were estimated to be $2.9 \%$ (average \pm 1 standard deviation) .

(2) Simulation of the experiment with JCDS-FX \& PHITS

To simulate the phantom experiments by JCDS-FX, a series of DICOM medical images of the phantom were taken by a CT device. For the CT data, the number of the slices was 60 , and the number of the pixels for a slice was 262,144 (512×512). The slice thickness was $3 \mathrm{~mm}$, and the pixel size was $0.5 \mathrm{~mm}$. A three-dimensional model of the phantom was created based on the CT images using JCDS-FX, and then several voxel calculation model described as below were configured from the 3D model.

(a) Material mixed voxel model: The models were created by using conventional voxel modeling method. The voxel size of the model was set as $2 \times 2 \times 2 \mathrm{~mm}^{3}$. The condition of the voxel model is applied to treatment planning of BNCT performed at JRR-4 at present. For the material definition for the voxel cell, mixed composition was set, when two or more materials were mixed in a voxel cell. Figure 4 shows cross sections view of the cylindrical water phantom model constructed by the material mixed model.

(b) Pixel based voxel model: all pixels were converted into miniature voxel cells directly. This model is ultimate precise geometry model. The number of all voxel cells was 15,728,640 $(512 \times 512$ pixels $\times 60$ Slices $)$. Figure 5 shows the pixel based voxel of the phantom.

(c) Uneven voxel model: by using the MLV method, the pixel based model was converted into a voxel model mixing different voxels sizes. Figure 6 shows the voxel model constructed by the MLV method. The number of all voxel cells was 39,000 (650 voxels per slice $\times 60$ slices). The air region and water region inside the phantom except for the boundary 


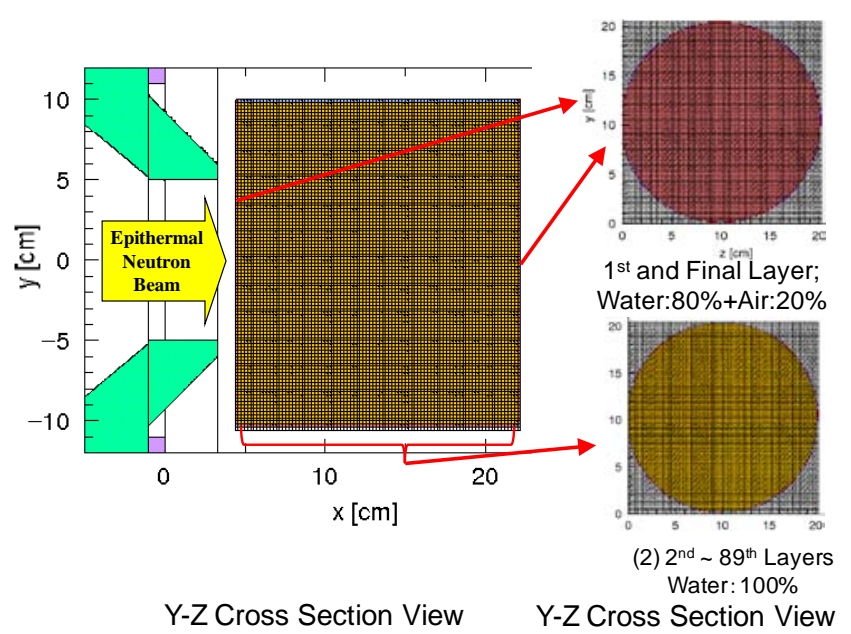

Fig. 4 Material mixed voxel model with $5 \times 5 \times 5 \mathrm{~mm}^{3}$ voxel

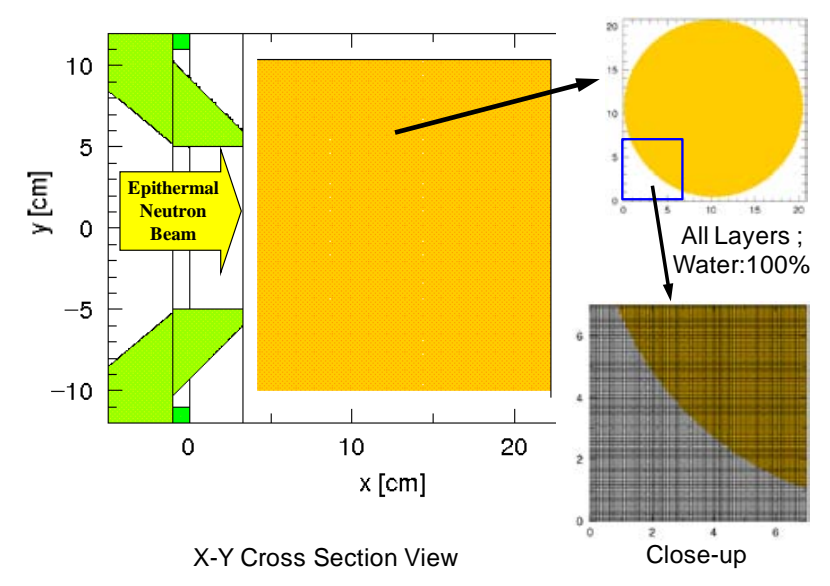

Y-Z Cross Section View

Fig. 5 Pixel based voxel model of the phantom

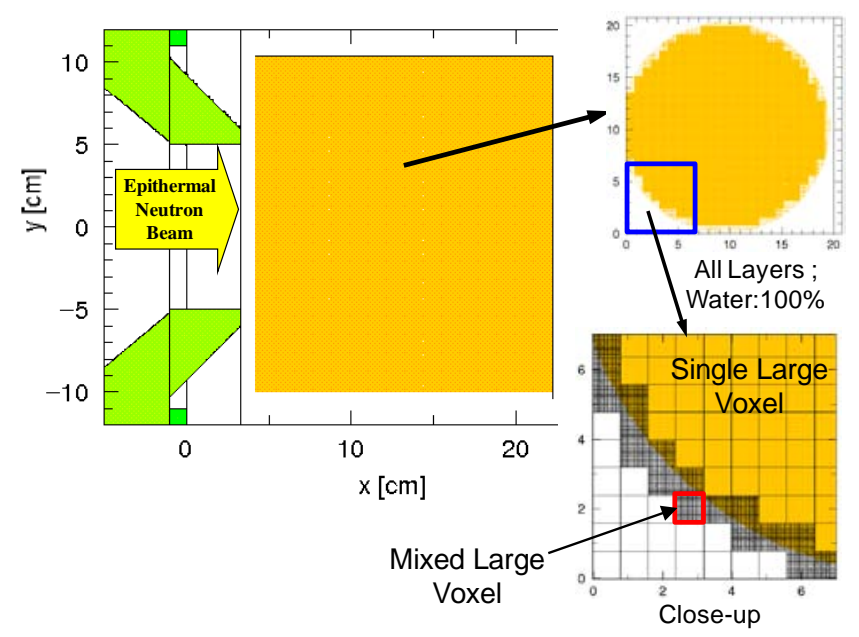

$X-Y$ Cross Section View

Y-Z Cross Section View

Fig. 6 Uneven voxel model created by the MLV method

region were constructed by the single large voxel, and the boundary region between air and phantom were configured by the mixed large voxel consisting of the pixel based voxel cells, as shown in the Fig. 6.

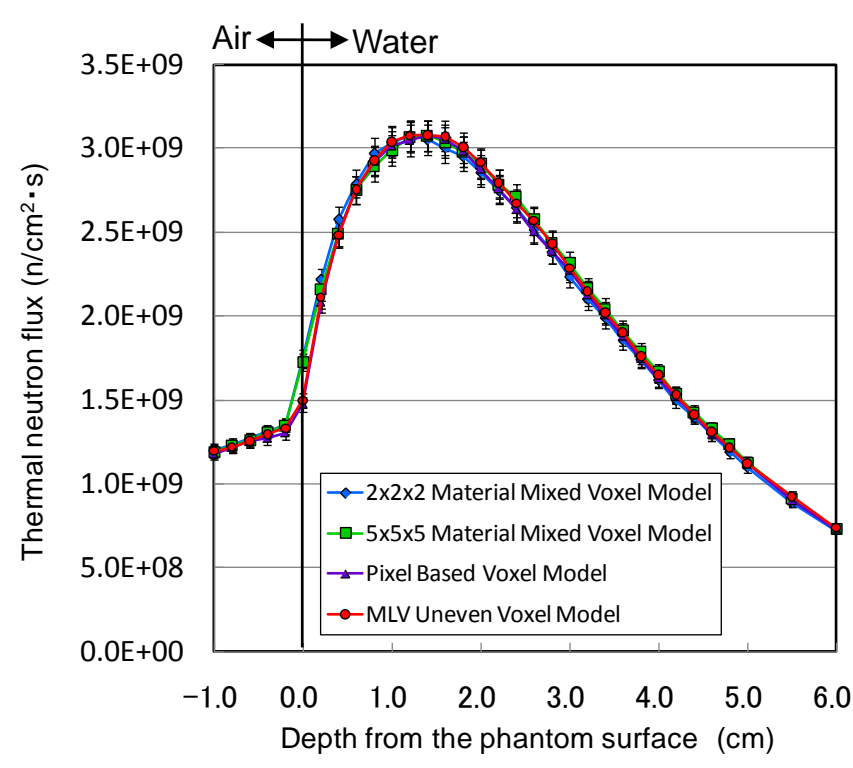

Fig. 7 Thermal neutron flux profiles for each voxel model

In the verification, with each calculation model, distribution of the thermal neutron flux $(\sim 0.53 \mathrm{eV})$ under the irradiation condition was determined, respectively. In the verification, PHITS was applied to every calculation. And the PHITS calculation was done by using conventional PC-Workstation (Intel Xeon Quad Core $2.4 \mathrm{GHz}$, RAM: $16 \mathrm{~GB}$, single core calculation).

To standardize the tally condition, distributions for the neutron fluxes and the dose rates were determined by "Mesh Tally". To compare the calculation performance with difference of the geometry, the region of the mesh tally was defined to determine the distributions in entire field of the phantom and then a mesh size was fixed as $2 \times 2 \times 2 \mathrm{~mm}^{3}$.

\section{Results and Discussions}

First, to confirm the calculation accuracy of the uneven voxel model, calculation results determined from each voxel model were compared. Figure 7 shows thermal neutron flux profiles on beam's central axis in the phantom for each voxel model. The statistical uncertainties of the calculated values for the each calculation were below 3\%. The calculated thermal neutron flux was normalized to the $3,500 \mathrm{~kW}$ reactor power as the reactor condition in the phantom experiment.

The thermal neutron fluxes determined by the uneven voxel model were corresponded to the values with the pixel based voxel model within the statistical errors throughout the estimation field. It stands to reason that values of the uneven model corresponds to the pixel based model's one because the geometry (material configuration for each miniature voxel cell) constructed by the MLV method is equal to the geometry of the pixel based model. And also the both calculated profiles were in good agreement with the experimental values measured by the phantom experiment. On the other hand, for the calculations with the material mixed voxel models, the profiles in the phantom deeper than $5 \mathrm{~mm}$ were comparable to the both profiles of the pixel based model and the uneven voxel model, with error of less than $3 \%$. Howev- 
Table 1 Calculation time for each voxel model

\begin{tabular}{cccc}
\hline Voxel Model & $\begin{array}{c}\text { Calculation } \\
\text { Time } \\
\text { (min) }\end{array}$ & $\begin{array}{c}\text { Ratio to } \\
\text { material } \\
\text { mixed } \\
\text { model }\end{array}$ & $\begin{array}{c}\text { Ratio to } \\
\text { pixel } \\
\text { based } \\
\text { model }\end{array}$ \\
\hline $\begin{array}{c}\text { Material Mixed } \\
\text { Voxel model }\end{array}$ & 30.9 & 1.0 & 0.41 \\
\hline $\begin{array}{c}\text { Pixel Based Voxel } \\
\text { model }\end{array}$ & 76.1 & 2.46 & 1.0 \\
\hline $\begin{array}{c}\text { Uneven Voxel } \\
\text { model with MLV }\end{array}$ & 36.1 & 1.17 & 0.47 \\
\hline
\end{tabular}

er, the calculation values of the material mixed voxel models at around the phantom surface were approximately $6 \%$ to $17 \%$ higher than the experimental value and the calculations for both of the minute voxel models. The discrepancy of the material mixed model caused by sharp building-up effect of thermal neutron which is occurred at surface region. It is difficult to estimate accurately the sharp distribution change by the calculation with the material mixed voxel model, the characteristic discrepancy at the boundary region between air and tissue region is pointed out many times before. ${ }^{7,15)}$ The results demonstrated that the MLV method allowed to perform high accuracy dose calculation equal to the calculation with the pixel based voxel model.

Next, the calculation speed of the uneven voxel model was evaluated by comparing with calculation times of the each calculation model. Table 1 shows the calculation time per 1 million particles calculation for the each voxel model. And ratio of the calculation time with the conventional material mixed voxel model and with the pixel based voxel model are also shown in the table, respectively.

By using the uneven voxel model, the calculation time was shortened about $50 \%$ against the pixel based voxel model. The results proved that the MLV method enabled the precise voxel model to reduce calculation time substantially while maintaining the high-accuracy in dose estimation. By comparison, calculation time of the uneven voxel model was about 1.2 times longer than the material mixed model calculation. However the calculation with the material mixed voxel model causes the characteristic discrepancy at boundary region between air and the phantom surface, as described above. By using the MLV method, raising the discrepancy can be suppressed. In the estimation for the cylindrical water phantom geometry, the results demonstrate practicality of the uneven voxel model's calculation with the MLV method. However, when the geometry is change from simple model like the water phantom to complicated human body, the calculation time of the uneven voxel model might become to be long with the conventional material mixed voxel model due to increase of the number of the mixed large voxel cell that forms the uneven voxel model. Thus to confirm effectiveness of the MLV method in actual treatment planning, dose estimation for geometries of several human bodies is being also carried out.

Furthermore at present, we are improving the algorithm further to make a calculation model for human body more efficient. For example, in the producing process of the single large voxel, region of the single large voxel changes to expanding as $16 \times 16 \times 2$ slice (512 pixels). By this way, the number of the voxel cells that forms an uneven voxel model will reduce further, the particle transport of the Monte-Carlo calculation is expected to become more efficient.

\section{Conclusion}

JCDS-FX with PHITS, a new multi-modal Monte-Carlo treatment planning system is being developed. The system allows making a precise voxel model consisting of pixel based voxel cells in order to perform high-accuracy dose estimation. However, the miniaturization of the voxel cell size causes calculation time to increase. Thus, to make particle transport in the Monte-Carlo calculation more efficient, the Multistep Lattice-Voxel Method which can construct a voxel model that combines different voxel sizes was devised. The method configures an uneven voxel model by utilizing the lattice function over and over. To verify the performance of the method, several calculations for a cylindrical water phantom geometry were carried out. The verification results proved that the method enabled the precise voxel model to reduce calculation time substantially while maintaining the high-accuracy in dose estimation. Several verifications with complicated human geometry are being performed at present. And we are still carrying out sophistication of the modeling method to work toward practical use of the new treatment planning system with more precise computational dosimetry.

\section{References}

1) L. E. Farr, W. H. Sweet, J. S. Robertson, C. G. Foster, H. B. Locksley, L. Sutherland, M. L. Mendelsohn et al., "Neutron capture therapy with boron in the treatment of gliobrastoma multiforme,” Am. J. Roentgnol., 71, 279-93 (1954).

2) L. E. Farr, W. Haymaker, T. Konikowski, S. W. Lippincott, "Effects of alpha particles randomly induced in the brain in the neutron-capture treatment of intracranial neoplasm," Int. J. Neurol. 3, 564-76 (1962).

3) Y. Nakagawa, K. Pooh, T. Kobayashi, S. Uyama, A. Matsumura, H, Kumada, "Clinical review of the Japanese experience with boron neutron capture therapy and a proposed strategy using epithermal neutron beams, “ J. Neurooncol., 62, 87-99 (2003).

4) T. Yamamoto, A. Matsumura, K. Nakaia, Y. Shibataa, K. Endoa, F. Sakurai, T. Kishi, H. Kumada, K. Yamamoto, Y. Torii "Current clinical results of the Tsukuba BNCT trial," Appl. Radiat. Isot., 61, 1081-1093 (2004).

5) I. Kato, Y. Fujita, A. Maruhashi, H. Kumada et al., "Effectiveness of BNCT for recurrent head and neck malignancies," Appl. Radiat. Isot., 67, 37-42 (2009).

6) H. Kumada, K. Yamamoto, A. Matsumura at el., "Development of JCDS, a computational dosimetry system at JAEA for boron neutron capture therapy,” J. Physics: Conf. Series, 74, 1-7 (2007).

7) H. Kumada, K. Yamamoto, A. Matsumura, T. Yamamoto, Y. Nakagawa, K. Nakai, T. Kageji, "Verification of the Computational Dosimetry System in JAERI (JCDS) for boron neutron capture therapy ," Phys. Med. Biol. 49, 3353-65 (2004).

8) S. Kawabata, S. Miyatake, T. Kuroiwa et al., "Boron Neutron 
Capture Therapy for Newly Diagnosed Glioblastoma,” J. Radiat. Res., 50, 51-60 (2009).

9) M. Umezawa, H. Sakurabata, M. Tadokoro, H. Ootsuka, H. Nishiuchi, K. Saito, K. Matsuda et al., "Beam commissioning of the new proton therapy system for University of Tsukuba," Proc. of Particle Accelerator Conference, 648-650 (2001).

10) H. Kumada, T. Nakamura, M. Komeda, A. Matsumura, "Development of a new multi-modal Monte-Carlo radiotherapy planning system,” Appl. Radiat. Isot., 67, 118-121 (2009).

11) H. Kumada, T. Nakamura, M. Komeda, A. Matsumura, "Development of a multi-modal Monte-Carlo radiation treatment planning system combined with PHITS," LASER-DRIVEN RELATIVISTIC PLASMAS APPLIED TO SCIENCE, INDUSTRY AND MEDICINE: 2nd International Symposium,
AIP Conference Proceedings, 1153, 377-387 (2009).

12) J. F. Briesmeister, MCNP-A General Monte Carlo Code $N$-Particle Transport Code Version 4C, LA-13709-M, Los Alamos National Laboratory (LANL) (2000).

13) H. Iwase, K. Niita, T. Nakamura, "Development of General-Purpose Particle and Heavy Ion Transport Monte Carlo Code,” J. Nucl. Sci. Technol., 39, 1142-1151 (2002).

14) K. Arigane, T. Yamada, D. Nemoto, M. Banba, K. Kawarai, Installation of JRR-2 Neutron Medical Irradiation Facility, JAERI-M 91-39, Japan Atomic Energy Research Institute (JAERI) (1991).

15) T. Seppälä, FiR-1 epithermal neutron beam model and dose calculation for treatment planning in neutron capture therapy, Report Series in Physics, HU-P-D103, University of Helsinki (2002). 